\title{
Das Richtige tun
}

\section{Michael Stamm}

Dr. med., D.E.A.A., Facharzt für Anästhesiologie und Intensivmedizin, EMBA Universität Zürich, Mitglied der FMH

In der Schweiz haben in den vergangenen 15 Jahren Überlegungen zur Abgeltung medizinischer Leistungen die Gesundheitsökonomie, die Politik und die Gesellschaft intensiv beschäftigt. Neue Finanzierungssysteme wurden eingeführt (2004 TARMED für die Abgeltung ambulanter Leistungen, 2012 swissDRG für stationäre Leistungen), und deren günstige und ungünstige Auswirkungen wurden sichtbar. Es wurden Fehlentwicklungen und Fehlanreize identifiziert, die sich daraus ergeben haben [1]. Aktuell, 2017, werden diese Fehlentwicklungen in der breiten Öffentlichkeit wahrgenommen. Es vergeht keine Woche, in der nicht gesundheitsökonomische Fragen in den Medien diskutiert werden, oft bemerkenswert differenziert. Politikern wird klar, dass der längst fällige Handlungsbedarf bezüglich dieser Fehlentwicklungen nicht mehr ignoriert werden kann. Mehr noch: Die Akteure im Gesundheitswesen insbesondere Leistungserbringer, Versicherer, kantonale Behörden - erkennen, dass sich auch im Gesundheitswesen der Schweiz disruptive Veränderungen anbahnen. Ein wichtiger Treiber dieser Veränderungen ist unsere informierte und befähigte Wissensgesellschaft, die an der Bearbeitung gesundheitspolitischer und medizinischer Fragestellungen partizipiert.

In dieser Situation stellt sich die Frage, wie sich Ärztinnen und Ärzte sowie die sie repräsentierenden Institutionen (nationale, kantonale und fachliche Ärztegesellschaften, aber auch Spitäler, Klinikketten, Ärztenetzwerke) verhalten sollen. Es erscheint sinnvoll und notwendig, in dieser Situation Prinzipielles zu bedenken.

\section{1. Ärztinnen und Ärzte sollten bei ihren Über-} legungen und Entscheidungen das Wohl der Patientinnen und Patienten über alles stellen. Dies nicht nur aufgrund des Berufsethos, sondern weil die Gesellschaft dies von ihnen erwartet und darauf vertraut, dass Ärztinnen und Ärzte dies tun. Wer sonst wenn nicht sie? In der heutigen Zeit bedeutet das für Ärztinnen und Ärzte insbesondere auch, den sogenann-

\section{Résumé}

Le système de santé publique de la Suisse se trouve devant des changements perturbants, avant tout à cause des changements de l'indemnisation des prestations. Quel rôle jouent les médecins? Comment doivent-ils se comporter? Des réponses en 7 principes. ten 'ePatients' - 'e' steht für 'equipped', 'enabled' oder auch 'empowered' - auf Augenhöhe zu begegnen und ihnen als neutraler Partner nach bestem Wissen und Gewissen beratend zur Seite zu stehen.

\section{2. Ärztinnen und Ärzte sollten transparent sein.} Sie sollten ihre Handlungsmotive und die grundlegenden Anreizsituationen offen darlegen. Sie sollten beispielsweise transparent machen, dass eine medizinische Behandlung deshalb stationär statt ambulant durchgeführt wird, weil sie bei stationärer Durchführung zum Teil mehrfach besser abgegolten wird.

Es ist absolut nachvollziehbar, wenn sich Leistungserbringer unter einer gegebenen Anreizsituation ökonomisch vernünftig verhalten. Es ist nicht in Ordnung, dafür einzelne Ärztinnen und Ärzte zu schelten. Es ist notwendig, falsche Anreizsituationen offen darzulegen, speziell durch Ärztinnen und Ärzte: Besonders sie sind in der Position, diese erkennen zu können. Nur dann können falsche Anreizsituationen korrigiert und menschlicher und wirtschaftlicher Schaden verhindert werden.

\section{3. Ärztinnen und Ärzte sollten andere Leute nicht täuschen.}

Beispielsweise sollte eine medizinische Massnahme nicht als notwendig hingestellt werden, wenn es nur darum geht, einen finanziellen Nutzen zu ziehen - wie die oben erwähnte Übernachtung im Spital, die nicht medizinisch oder aus Gründen der Betreuung angezeigt ist. Noch deutlicher formuliert: Man sollte die ärztliche Autorität nicht dafür missbrauchen, um die Leute hinters Licht zu führen. Dies ist nicht nur verwerflich und gesetzeswidrig (im obigen Beispiel: Missachtung der WZW-Kriterien des KVG). Es schadet auch dem ärztlichen Stand, weil es seine Glaubwürdigkeit untergräbt.

\section{4. Ärztinnen und Ärzte sollten die Gesellschaft} von den Früchten des medizinischen Fortschritts profitieren lassen.

Fortschrittsbedingte Einsparungen sollen an jene zurückgeben werden, die die Einsparungen möglich gemacht haben. In erster Linie ist dies die Gesellschaft (ökonomisch repräsentiert durch Steuer- und Prämienzahler), welche medizinische Fakultäten und Forschungseinrichtungen finanziert. Beispielsweise ist es 
nicht mehr gerechtfertigt, eine Katarakt-Operation, die heute dank des medizinischen Fortschritts risikoarm in 8-10 Minuten fliessbandmässig durchgeführt werden kann - statt in 45-60 Minuten wie noch bei der Konzeption des Tarmed-Tarifs in den 90er-Jahren des letzten Jahrhunderts -, mit 2300 Franken oder mehr abzugelten. Weniger risikoreich, weniger zeitlicher (d.h. personeller) Aufwand, geringerer Bedarf an anästhesiologischen Leistungen: Von diesen Effekten des medizinischen Fortschritts soll auch die Gesellschaft finanziell profitieren, nicht nur die einzelne Ärztin / der einzelne Arzt.

Es geht darum, die eigene Rolle nicht zu missbrauchen, sondern vertrauensbildend, selbstbewusst und mit Gemeinsinn wahrzunehmen.

5. Ärztinnen und Ärzte sollten sich gegen ungerechtfertigte und sich unwirtschaftlich auswirkende Rückstufungen der Abgeltung ihrer Leistungen wehren.

Gewinn, Überschuss zu erwirtschaften ist für alle ein Erfordernis, damit die Zukunft selbstbestimmt gestaltet werden kann. Dies gilt nicht nur für Ärzte, ist aber gerade für diese unerlässlich, weil sie oft selbständig unternehmerisch tätig sind und unternehmerische Risiken eingehen. Sich gegen ungerechtfertigte und sich unwirtschaftlich auswirkende Rückstufungen der Abgeltung zu wehren ist auch geboten, um die Versuchung tief zu halten, die Lücken eines Abgeltungssystems systematisch auszunutzen.

\section{6. Ärztinnen und Ärzte sollten sich nicht scheuen, einen anständigen und gerechten Verdienst einzufordern.}

Es ist nicht nur aus unternehmerischen Gründen und aus Gründen des Erhalts der Versorgungssicherheit wichtig, sich in einem Umfeld mit streng regulierten Abgeltungsmodalitäten gegen ungerechtfertigte und sich unwirtschaftlich auswirkende Rückstufungen der Abgeltung zu wehren. Lachhaft tiefe Abgeltungen sind auch der Qualität der Leistungserbringung abträglich. Und sie werden als herabwürdigend und Respektlosigkeit empfunden. Beispielsweise ist eine ärztliche Entschädigung (AL im TARMED-Tarif) für eine Carpaltunnel-Operation von 82.14 Taxpunkten (im Kanton Bern CHF 70.65 entsprechend) gemäss aktuellen bundesrätlichen Revisionsvorschlägen nicht nur nicht adäquat, sondern ein Hohn, vergleicht man diese Abgeltung mit jenen in der Finanzbranche, in der Informatikbranche oder nur schon mit dem Handwerk. Gut zu verdienen ist eine angemessene Form der Abgeltung von hohem zeitlichem, intellektuellem und menschlichem Engagement und von der Bereitschaft, Risiken der Berufsausübung verantwortungsvoll zu übernehmen. Als Absolvent eines Medizinstudiums und einer spezialärztlich Weiterbildung hat man die Bereitschaft zu diesem Engagement und zu verantwortungsvollen Risikonahmen bewiesen. Man hat auch einige Jahre auf Verdienst verzichtet. Es ist legitim, diesen in einer verkürzten Erwerbstätigkeitsspanne kompensierend erwirtschaften zu können.

\section{7. Ärztinnen und Ärzte sollten einfordern und} durchsetzen, dass ihre Sach- und Fachkompetenz berücksichtigt wird.

Sie sollten das Spielfeld nicht alleine Gesundheitsökonomen, Gesundheitspolitikern und Managern ohne medizinischen Sachverstand überlassen. Dies ist noch besser möglich, wenn sich Ärztinnen und Ärzte Kenntnisse betriebswirtschaftlicher und gesundheitspolitischer Zusammenhänge aneignen.

\section{Wozu diese Prinzipien?}

Die absehbaren Veränderungen, insbesondere der Abgeltung medizinischer Leistungen, haben das Potenzial, das Schweizerische Gesundheitswesen von Grund auf zu verändern. Wird beispielsweise eine einheitliche Finanzierung ambulanter und stationärer Leistungen (EFAS) eingeführt, werden ambulante Behandlungen nicht mehr verhindert. Dies wird dazu führen, dass herkömmliche Spitalinfrastrukturen und -prozesse nur noch für schätzungsweise 30-40\% der durchzuführenden Operationen benötigt werden und nicht mehr wie heute für $80 \%$. Eine bessere Ausrichtung auf die Bedürfnisse von Patientinnen und Patienten, enorme Redimensionierungen bei Investitionen in medizinische Infrastrukturen sowie ein effektiverer Einsatz der im Gesundheitswesen beschäftigten Personen [2] werden ermöglicht. Das Gesundheitswesen braucht Ärztinnen und Ärzte, die diese anstehenden Veränderungen integer, vertrauenswürdig und kompetent begleiten und mitgestalten. Es geht darum, die eigene Rolle nicht zu missbrauchen, sondern vertrauensbildend, selbstbewusst und mit Gemeinsinn wahrzunehmen. Dies ist für das Gemeinwesen und für die Profession wichtig. Ärztinnen und Ärzte spielen in unserer Gesellschaft und in unserem Gesundheitssystem eine umso gewichtigere Rolle, je konsequenter die genannten Prinzipien beachtet werden. 